\title{
Mediastinal debulking for a T-cell leukaemia/lymphoma presenting with cardiac tamponade
}

\author{
Sotirios D. Moraitis ${ }^{1}$, Apostolos C. Agrafiotis ${ }^{1,2}$, Leonidas Marinos ${ }^{3}$, Ioannis Panagiotou1, \\ Timotheos Sakellaridis $^{4}$, Christos Tsakalakis ${ }^{5}$, Elias Poulakidas ${ }^{6}$ \\ ${ }^{1}$ Joint Corps Armed Forces Cardiac Surgery Department, 401 Hellenic Army Hospital, Athens, Greece; ${ }^{2}$ Department of \\ Thoracic Surgery, Saint-Pierre University Hospital, Université Libre de Bruxelles, Brussels, Belgium; ${ }^{3}$ Department of \\ Pathology, Evangelismos General Hospital, Athens, Greece; ${ }^{4}$ Department of Thoracic Surgery, 401 Hellenic Army \\ Hospital, Athens, Greece; ${ }^{5}$ Intensive Care Unit, 401 Hellenic Army Hospital, Athens, Greece; ${ }^{6}$ Department of \\ Haematology, 401 Hellenic Army Hospital, Athens, Greece
}

\begin{abstract}
Anterior mediastinal masses are relatively uncommon and include a wide variety of lesions. Lymphomas account for $25 \%$ of anterior mediastinal masses. Lymphomas and other haematological malignancies are associated with pericardial effusion. There are also cases where a cardiac tamponade occurred. The aim of the case reported herein is to discuss the surgical approach and particularly the mediastinal debulking as an adjunct to systematic treatment for haematological diseases presenting as an anterior mediastinal mass responsible for a cardiac tamponade.
\end{abstract}

\section{Introduction}

Anterior mediastinal masses are relatively uncommon and include a wide variety of lesions. Thymic lesions are the most frequent $(35 \%)$ whereas lymphomas account for $25 \%$ of anterior mediastinal masses (Hodgkin's disease 13\% and non-Hodgkin

Correspondence: Dr. Apostolos Agrafiotis, Department of Thoracic Surgery, Saint-Pierre University Hospital, Rue Haute 322, 1000 Brussels, Belgium.

E-mail: apostolos_agrafiotis@stpierre-bru.be

Key words: Mediastinum; debulking; lymphoma; tamponade.

Contributions: The authors contributed equally.

Conflict of interest: The authors have no conflict of interest to declare.

Received for publication: 25 March 2019.

Accepted for publication: 9 May 2019.

(C) Copyright S.D. Moraitis et al., 2019

Licensee PAGEPress, Italy

Monaldi Archives for Chest Disease 2019; 89:1072

doi: 10.4081/monaldi.2019.1072

This article is distributed under the terms of the Creative Commons Attribution Noncommercial License (by-nc 4.0) which permits any noncommercial use, distribution, and reproduction in any medium, provided the original author(s) and source are credited. lymphoma 12\%) [1]. Lymphomas occur in the mediastinum as a part of a disseminated disease or as a primary lesion [2]. Lymphomas and other haematological malignancies are associated with pericardial effusion. There are also cases where a cardiac tamponade occurred [3-11]. The aim of the case reported herein is to discuss the surgical approach and particularly the mediastinal debulking as an adjunct to systematic treatment for haematological diseases presenting as an anterior mediastinal mass responsible for a cardiac tamponade.

\section{Case report}

A 24-year old male patient with no past medical history presented to the Emergency Department complaining of a progressively installed shortness of breath within some weeks. The dyspnoea became worse during the last three days.

A chest X-ray (Figure 1) showed a mediastinal enlargement. A chest $\mathrm{CT}$ scan revealed an anterior mediastinal mass together with a pericardial effusion (Figure 1). The biological markers (afp, LDH, $\beta$-hCG) were within normal limits. A percutaneous CTguided needle biopsy was scheduled for the next day. However, the clinical state of the patient rapidly deteriorated over the next few hours. A cardiac ultrasound examination showed signs of cardiac tamponade.

The patient was rapidly admitted to the OR for emergency pericardial drainage. The CT scan analysis showed that there was no space for a subxiphoidal approach because of the bulky tumour. For that reason, a wide pericardial window through a median sternotomy was preferred. Emergent surgical approach was preferred over percutaneous drainage for the same reason and in order to perform biopsies on the same time. After sternotomy, a bulky tumour was encountered, invading the pericardium from the diaphragm to the anonymous vein and between the two phrenic nerves. The opening of the pericardium permitted the evacuation of nearly 2 litres of serosanguinous fluid. Because of pericardial invasion and in order to perform a wide window, a mediastinal debulking was performed (Figure $2 \mathrm{a}, \mathrm{b}$ ). The haemodynamic state of the patient was rapidly stabilised. The postoperative course was uneventful and the patient was discharged on postoperative day 10 .

Histopathological analysis was compatible with an adult T-cell leukaemia/lymphoma (WHO classification of tumours of haematopoietic and lymphoid tissues 2017 9827/3. Immunohistochemical analysis was $\mathrm{Tdt}+, \mathrm{CD} 3+, \mathrm{CD} 4+, \mathrm{CD} 8+$ 
(CD4+ < CD8+), CD1a+, CD2+ CD7+, CD5+, CD79-, bcl-2-, CD45RO-, CD10-, CD20-, CD34-, MPO-, CD56-, bcl-6-, PAX-5(Figure 2c-f).

The patient was then referred to the haematology clinic were a chemotherapy protocol (German Multicenter Study Group for Adult Acute Lymphoblastic Leukemia-GMALL) was initiated [12]. The patient presented a complete response and follow-up after 18 months, showed no recurrence into the mediastinum (Figure 3).

\section{Discussion}

It is possible to distinguish anterior mediastinal masses based exclusively on demographic, clinical and radiological features, but still sometimes tissue biopsy is warranted in order to establish the diagnosis and guide the therapeutic strategy [1]. Pericardial effusion is encountered in 5\% of patients with Hodgkin's lymphoma and it is usually asymptomatic [3]. Nevertheless, pericardial effusion as an initial presentation is quite rare [3]. Maisch et al., reported that in 42 patients with cancer who developed pericardial effusion, $4.8 \%$ suffered a Hodgkin's disease [4]. Symptomatic pericardial effusion and even cardiac tamponade could be the first manifestation of an occult malignancy [4,5]. Patterns of cardiac and pericardial involvement from haematological malignancies include retrograde lymphatic spread, haematogenous spread and direct invasion of a mediastinal mass $[2,3,6,7]$. Pericardial effusion in not a sine qua non condition since massive pericardial invasion by the tumour can lead to a tamponade physiology [8]. Pericardiocentesis is usually performed for diagnostic reasons and as initial treatment of tamponade in order to improve haemodynamic instability. However, the cytological examination of the pericardial fluid cannot always establish the diagnosis, since the effusion can be reac-
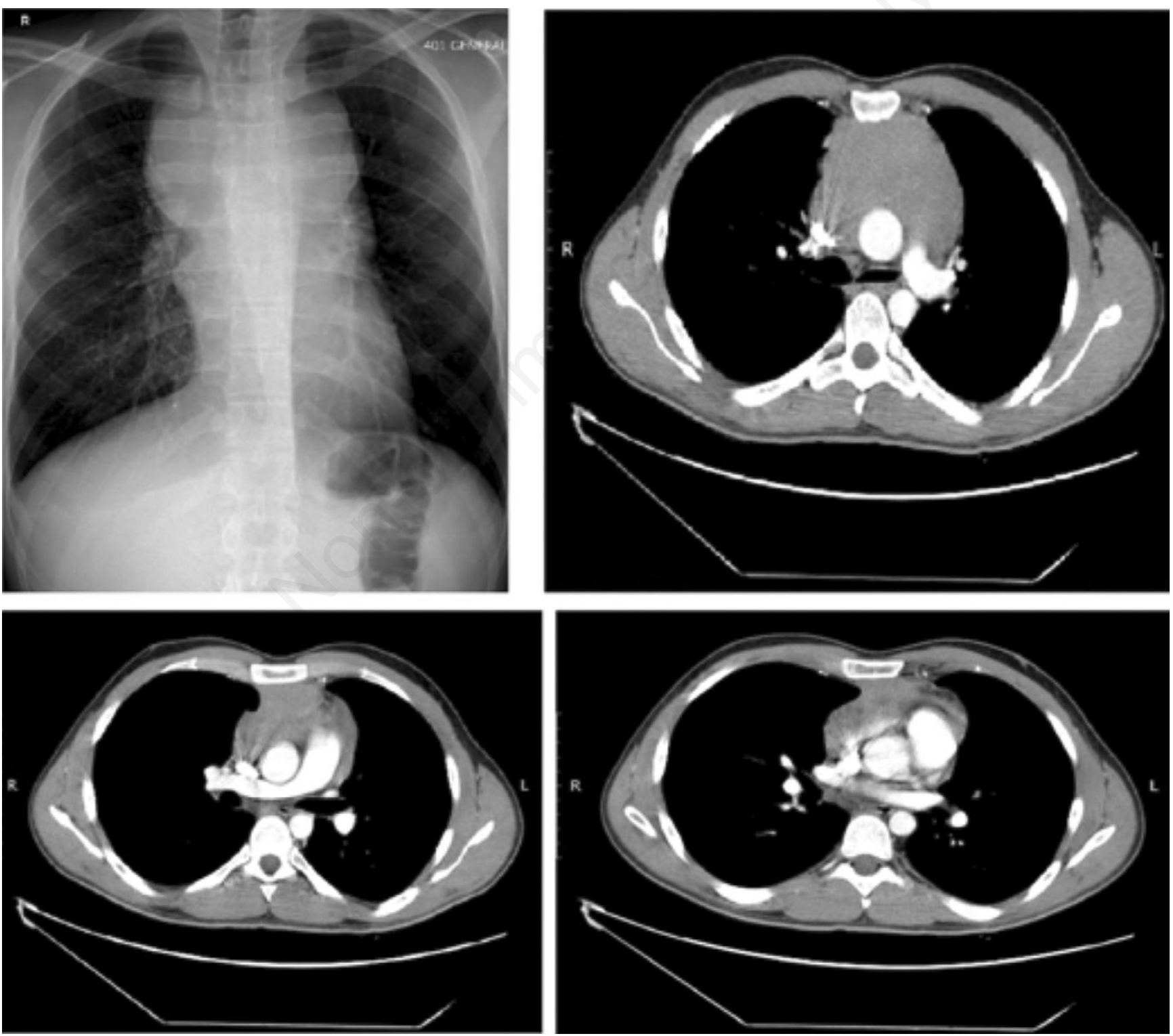

Figure 1. Preoperative chest $\mathrm{x}$-ray and CT scan. 
tive [6]. In the majority of case reports a systematic treatment (chemotherapy, radiotherapy, targeted therapy, allogenic stem cell transplantation) was administrated [6,7,9-11].

Ricci et al. performed mediastinal debulking in 5 patients with isolated mediastinal lymphoma [13]. Three patients were alive after 5 to 11 years and 2 two died after 36 and 40 months. They propose that mediastinal debulking might be considered in selected cases in order to enhance long-term results.

There is evidence concerning debulking surgery in the context of Castleman's disease. Talat et al. [14] conducted a systematic review; mediastinal debulking was described in $77 \%$ of all patients and it was more common in unicentric than multicentric disease. In multicentric disease outcomes were comparable between debulk- ing surgery alone, immunochemotherapy alone or a combination of both. They also concluded that the role of debulking surgery in human immunodeficiency virus (-) multicentric Castleman's disease needs to be evaluated in prospective studies [14].

Nagano and Matsumoto [15] reported the debulking surgery of an anterior mediastinal mass due to the lack of diagnosis, even after percutaneous biopsy, and because of the clinical deterioration of the patient. The histological analysis revealed a Castleman's disease; after a transient improvement of half a year, there was a recurrence in the cervical lymph nodes and the patient was treated with corticosteroids [15].

In the case reported herein a mediastinal debulking was performed not in a curative intent but in order to perform a wide
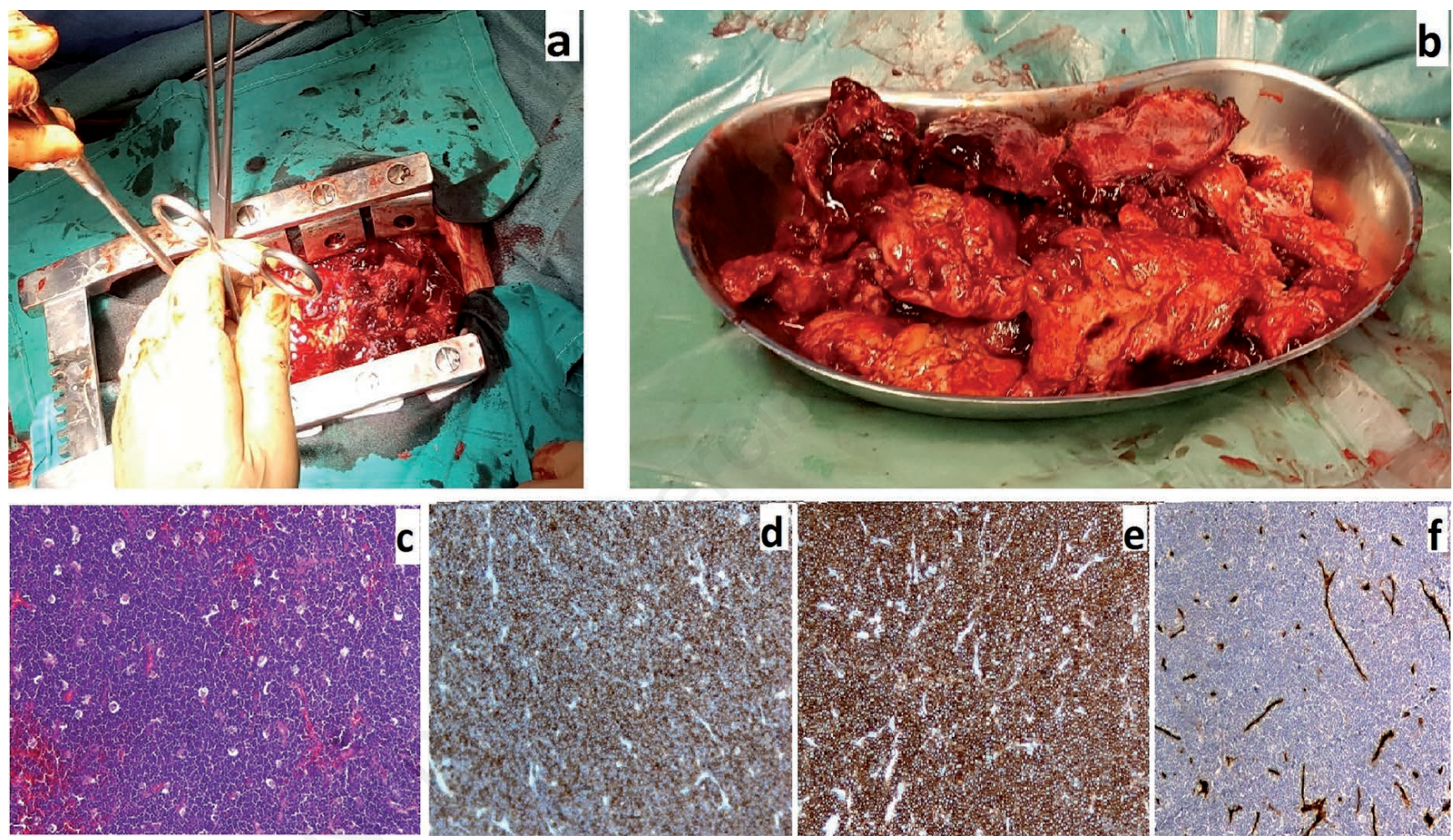

Figure 2. a) Perioperative image during mediastinal debulking. b) The resected specimen. c) Histopathological image (H\&E $x 20)$ showing a diffuse infiltration from medium-sized immature lymphocytes. d) Immunohistochemistry positive for CD3. e) Immunohistochemistry positive for CD8. f) Absence of immunohistochemical expression for CD34.
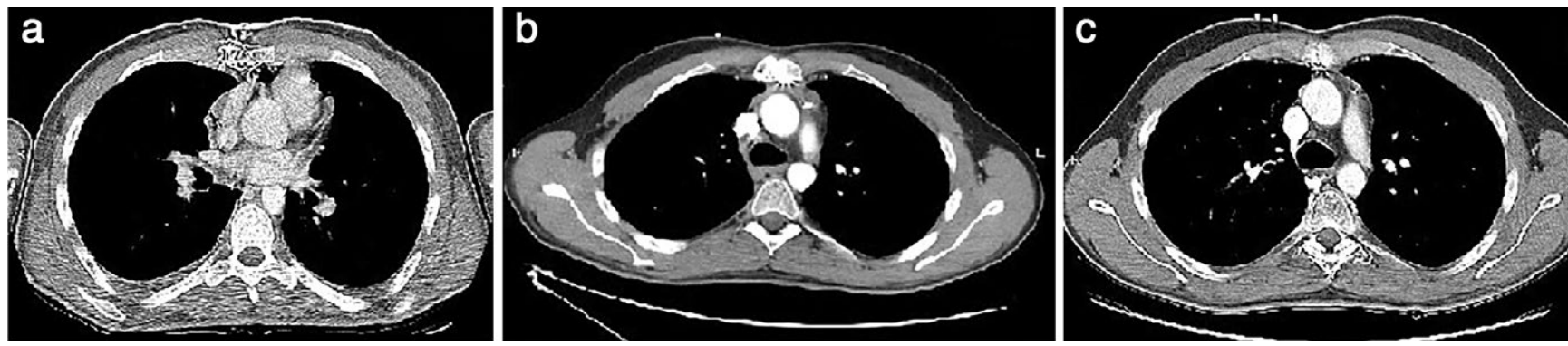

Figure 3. Postoperative CT scans at 1 month (a), 6 months (b) and 12 months (c). 
pericardial window because of the absence of space for pericardiocentesis or subxiphoidal approach due to the bulky tumour. We do not know if the debulking contributed to the good evolution of the patient or on either way the patient could have been cured by the chemotherapy alone. On the contrary, we are certain that mediastinal debulking is a feasible, quick and reliable alternative for the first line treatment of cardiac tamponade in extreme cases when pericardiocentesis and subxiphoidal approach are difficult to perform.

\section{Conclusions}

In extreme cases of anterior mediastinal masses presenting with a cardiac tamponade and when pericardiocentesis and subxiphoidal approach are difficult to perform, mediastinal debulking is a feasible, rapid and reliable procedure in order to treat the hemodynamic instability, perform a wide pericardial window and obtain sufficient tissue for biopsy. It remains to be proven that mediastinal debulking can be part of a multimodality treatment in case of haematological malignancies, as in the case reported herein.

\section{References}

1. Carter BW, Marom EM, Detterbeck FC. Approaching the patient with an anterior mediastinal mass: a guide for clinicians. J Thorac Oncol 2014;9:S102-9.

2. Uwabe Y, Yoshida H, Onishi M, et al. Primary mediastinal diffuse large cell lymphoma initially presented with pericardial infiltration. Intern Med 1992;31:1387-91.

3. Hajra A, Bandyopadhyay D, Layek M, et al. Cardiac tampon- ade as initial presentation of Hodgkin lymphoma. J Clin Imaging Sci 2015:5;67.

4. Maisch B, Ristic A, Pankuweit S. Evaluation and management of pericardial effusion in patients with neoplastic disease. Prog Cardiovasc Dis 2010;53:157-63.

5. Perek B, Tomaszewska I, Stefaniak S, et al. Cardiac tamponade - unusual clinical manifestation of undiagnosed malignant neoplasm. Neoplasma 2016;63:601-6.

6. Muthusamy P, Ebrom S, Cohle SD, et al. Pericardial involvement as an initial presentation of anaplastic large cell lymphoma. Can Fam Physician 2014;60:638-41.

7. Leung WH, Tai YT, Lau CP, et al. Cardiac tamponade complicating leukaemia: immediate chemotherapy or pericardiocentesis? Postgrad Med J 1989;65:773-5.

8. Esfahani K. An unusual cause of STEMI and cardiac tamponade. BMJ Case Rep 2014. doi:10.1136/bcr-2014-206926.

9. Erdogan E, Bacaksiz A, Akkaya M, et al. An unusual presentation of lymphoma: chylotamponade. Heart Lung 2012;41:617-20.

10. Chaves FP, Quillen K, Xu D. Pericardial effusion: a rare presentation of adult T-cell leukemia/lymphoma. Am J Hematol 2004;77:381-3.

11. Kumar A, Puwada S. Mantle cell lymphoma presenting as cardiac tamponade. Blood 2015;126:1255.

12. Gökbuget N, Hoelzer D, Arnold R, et al. Treatment for adult ALL according to protocols of the German Multicenter Study Group for Adult ALL (GMALL). Hematol Oncol Clin North Am 2000;14:1307-25, ix.

13. Ricci C, Rendina EA, Venuta F, et al. Surgical approach to isolated mediastinal lymphoma. J Thorac Cardiovasc Surg 1990;99:691-5.

14. Talat N, Belgaumkar AP, Schulte KM. Surgery in Castleman's disease: a systematic review of 404 published cases. Ann Surg 2012;255:677-84.

15. Nagano M, Matsumoto J. A case of TAFRO syndrome with a large mediastinal mass treated with debulking surgery. Surg Case Rep 2016;2:61. 